Rev. salud pública. 14 sup (1): 68-83, 2012

\title{
Crisis y transformaciones del trabajo en el nuevo modelo de acumulación
}

\section{Job crisis and transformations in the new model of accumulation}

\author{
Álvaro Zerda-Sarmiento \\ Facultad de Economía. Universidad Nacional de Colombia. Bogotá. azerdas@unal.edu.co \\ Recibido el 15 de Octubre de 2009/Enviado para Modificación 4 Marzo 2010/Aceptado 11 Diciembre 2010
}

\section{RESUMEN}

La crisis generalizada y estructural por la que atraviesa el capitalismo mundial es la manifestación de las dificultades del modelo de acumulación que se comenzó a perfilar desde la década del 70 en los países desarrollados y que busca reacomodarse sobre la base de los planteamientos neoliberales y un nuevo paradigma tecno económico. El nuevo patrón de acumulación ha tenido profundos impactos en el mundo del trabajo, que se manifiestan en todos los componentes de las relaciones laborales. En Colombia, la adopción del modelo ha sido parcial y segmentada pero sus implicaciones y la crisis prolongada también han repercutido sobre los trabajadores bajo la forma de desempleo, precariedad, segmentación, informalidad y débil cobertura de la seguridad social privatizada, mientras la acumulación financiera recircula los excedentes del trabajo. El modelo que intenta montar el actual gobierno lleva a centrar la economía en el sector primario y hacer así mismo más precaria la situación de grandes masas de la población. Superar el actual estado de cosas demanda creatividad del mundo laboral, que deberá buscar nuevos esquemas para la expresión y movilización de sus reivindicaciones, presionando por el establecimiento de un modelo económico diferente para construir un futuro más incluyente y con justicia social.

Palabras Clave: Crisis económica, desarrollo económico, empleo, protección social, paradigma tecno económico (fuente: DeCS, BIREME)

\section{ABSTRACT}

The general and structural crisis capitalism is going through is the token of the difficulties accumulation model has been dealing with since 70's in developed countries. This model has been trying to settle down again on the basis of neoliberal principle and a new technical-economical paradigm. The new accumulation pattern has had a effect in employment sphere which have been made evident at all the elements that constitute work relationships. In Colombia, this model implementation has been partial and segmented. However, its consequences (and the long-term current crisis) have been evident in unemployment, precarious work, 
segmentation, informal work and restricted and private health insurance. Besides, financial accumulation makes labour profits flow at different levels. The economic model current government has aimed to implement leads to strengthening exports, so making population life conditions more difficult. In order to overcome the current state of affairs, the work sphere needs to become more creative. This creative approach should look for new schemes for expression and mobilization of work sphere's claims. This is supposed to be done by establishing a different economic model aimed to build a more inclusive future, with social justice.

Key Words: Economic crisis, economic growth, employment, social protection, technical-economical paradigm (source: Mesh)

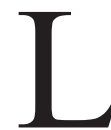

a mayoría de interpretaciones que se han hecho de la actual crisis económica la identifican como de naturaleza eminentemente financiera, resultado del comportamiento especulativo de algunos agentes económicos. En una dirección contraria, en el presente documento se caracteriza la crisis como de largo plazo y manifestación del reacomodo del modelo de acumulación, que replantea las relaciones entre el capital y el trabajo, con profundas repercusiones sobre las exigencias de desempeño a los trabajadores y en todo el espectro de las relaciones laborales. A partir de la descripción de ese panorama global, en el escrito se aborda el contexto de la realidad colombiana y las implicaciones sobre los componentes de seguridad social, para concluir con una reflexión en torno a los posibles elementos de una estrategia a seguir por los trabajadores para enfrentar la coyuntura con una visión que busque modificar la estructura del modelo de desarrollo económico y social en el país.

\section{CRISIS DEL MODELO DE ACUMULACIÓN}

La crisis por la que atraviesa el capitalismo mundial, considerada la mayor desde la Gran depresión de los años 30, es generalizada y estructural, en tanto tiene manifestaciones en los campos económico, ambiental, alimentario, energético y de los derechos humanos, pero además corresponde a un reacomodo en los patrones de acumulación, que desde las décadas de los ochenta del siglo XX se han venido ajustando por medio de un profundo cambio en sus bases tecnológicas, primero con la llamada revolución microelectrónica y en la actualidad con los desarrollos de la ingeniería genética y la nanotecnología (1). 
Características generales de la crisis actual

Como en anteriores crisis, la actual corresponde a un proceso de restructuración del modelo de acumulación que, en el marco de los límites mostrados por la práctica de la ideología neoliberal, busca un nuevo paradigma tecnoeconómico organizado sobre la base de las tecnologías de la información y las comunicaciones (TIC), alrededor de las llamadas ciencias de la vida y la nanotecnología, junto con un reacomodo, a lo largo del sistema financiero, de la apropiación de los flujos de excedentes económicos generados.

Es por esto que el papel protagónico de las instituciones financieras en la presente crisis no es gratuito ni ocasional, pero tampoco es la causa motivante de la misma, sino tan sólo la manifestación más prominente del estrangulamiento del circuito económico causado por la contención de los ingresos salariales adelantada desde la caída del muro de Berlín, que llevó a la generación de una gran burbuja crediticia para apalancar el consumo, la que finalmente estalló ante la debilidad de las innovaciones financieras surgidas merced a la desregulación mundial de la actividad del sector (1).

Transformaciones del modelo productivo.

El permanente reacomodo del sistema capitalista se ha dado en parte por medio de profundas transformaciones de su base material durante las décadas recientes: la importancia acrecentada del conocimiento como activo intangible, o capital vivo, constituye una novedosa fuente para la acumulación y la especulación que viabiliza los movimientos financieros. Es lo que algunos teóricos denominan el "capitalismo cognitivo" (2).

Los componentes más visibles del modelo productivo que se ha configurado durante las cuatro últimas décadas están dados, en primer lugar por la gran oleada de invenciones e innovaciones introducidas a partir del desarrollo de la microelectrónica que permitió el avance de la computación y su utilización como tecnología transversal a los procesos de producción de bienes y servicios y a la provisión de éstos a los mercados finales y su utilización en las TIC. Con base en ellas, se instauran los sistemas flexibles de producción, que dan origen a entidades con estructuras horizontales integradas, en donde se exige un mayor involucramiento del trabajador en la toma de decisiones y solución de problemas pero también permite 
desagregar el proceso en su conjunto, en lo que se dio en llamar outsourcing o subcontratación, que pasa por la relocalización de procesos, incluso en el plano internacional, verdadero componente de la aclamada globalización, existente tan solo para los capitales, los que entran en procesos de nueva competencia, estructurada como el surgimiento de diversos esquemas empresariales de colaboración en alianzas o coparticipación de riesgos (3).

En todo este proceso el conocimiento ha ganado papel como principal componente de la producción, lo cual no implica que en patrones anteriores de producción no fuera importante sino más bien que en su nueva configuración se convierte en protagónico, por encima del trabajo vivo, incluso. La batalla competitiva en el mercado pasa entonces por la utilización y el desarrollo de las modernas tecnologías y la permanente innovación, adoptada de manera estratégica como parte de la cultura corporativa y de relacionamiento frente al mundo laboral debilitado y derrotado en perspectiva histórica (4).

Interpretación de la actual crisis

Por todas estas nuevas configuraciones del modelo productivo, que llevan a un novedoso patrón de circulación y apropiación de los excedentes a través del sistema financiero (o financiarización) en lo que constituye un nuevo patrón de acumulación, para los observadores y analistas involucrados con la visión adscrita al sistema económico y formados dentro de un pensamiento económico ortodoxo, era difícil interpretar los hechos y encontrar elementos para anticipar los movimientos económicos por cuanto no podían identificar sus reales contornos ni la dirección de sus esguinces.

Así, entonces, era lógico que al presentarse la crisis también se revelara la incapacidad de la teoría para identificarla y más aun, para evitarla (1). Surgen desde luego preguntas que están en trance de ser resueltas por la dirección que tomen los acontecimientos y el papel que en ello asuman los movimientos sociales ¿Estamos frente a un nuevo tipo de capitalismo en ciernes? ¿O es ésta una nueva fase de acumulación basada en el mundo bioindustrial en proceso de gestación? Por ahora lo cierto es que el nuevo patrón productivo tiene profundas implicaciones sobre aquélla parte de la ecuación más débil, la de los trabajadores. 


\section{EL MUNDO DEL TRABAJO}

La modificación del modelo de acumulación implica cambio del paradigma tecnoeconómico, que tiene repercusiones sobre el mundo del trabajo, las que se manifiestan como nuevas exigencias al trabajador en cuanto a su desempeño en la fábrica, nuevas formas de las relaciones laborales, la incertidumbre frente a la posibilidad de conseguir empleo, su naturaleza y esquemas, el cubrimiento de su seguridad social y, con todo ello, la posibilidad de desarrollo en tanto ser humano.

Exigencias al trabajador

Desde la década de los 80 del siglo XX se ha evidenciado como una de las exigencias que las nuevas tecnologías hacen a los trabajadores la de polivalencia, como aquella capacidad de tener movilidad entre actividades y oficios diferentes, al interior de la planta, que viene a reemplazar la experiencia y especialización ganada por dedicación en el tiempo a una tarea $\mathrm{u}$ oficio. Esto incide en las calificaciones del trabajador y su capacidad para aplicar el pensamiento abstracto, con lo que reemplaza la experticia alcanzada con el tiempo. Con ello la noción tradicional de calificaciones es reemplazada por la de competencias, entendidas como el conjunto de habilidades generales y específicas que el trabajador debe poseer o adquirir para desempeñar cualquier posición en una organización (y hasta en la sociedad).

Así, la dedicación y precisión para realizar movimientos requeridos en la cadena de montaje por los puestos anterior y posterior a la ubicación del trabajador en la línea, es reforzada en el nuevo modelo por la habilidad para la comunicación, ya que se requiere de la participación del trabajador individual en grupos de trabajo que se organizan, coordinan, forman y desintegran de acuerdo con la necesidad de los procesos (3).

Los sistemas de trabajo también se han visto modificados, al punto de que se permite la actividad no presencial en el puesto de trabajo, en la modalidad conocida como teletrabajo -o trabajo a distancia- que puede desempeñarse desde el hogar o cualquier otro punto fuera de la empresa, reemplazando así la interacción entre los trabajadores. También nuevas maneras de organizar los procesos en donde, por ejemplo, los call centers o centros de llamadas configuran nuevas formas de ocupación (5). 
Relaciones laborales

Los mayores cambios en el campo de las relaciones laborales tienen que ver con transformaciones en las relaciones de empleo y las relaciones de trabajo bajo una nueva forma flexible, tanto numérica (rápido ajuste de la cantidad de trabajadores, sea mediante el despido, la interrupción temporal de los contratos o formas de contratación intermitentes) como funcional (diversas calificaciones de la fuerza de trabajo, desempeño de tareas variadas y formas de pago en diferentes modalidades).

Relaciones de trabajo. En diversos estudios (5) ha sido señalada la insistencia en la necesidad de un cambio cultural en las organizaciones, dirigido hacia la toma en conciencia de un conjunto de valores que se introyecten en los individuos y tengan efectos visibles en su comportamiento de fidelidad con la empresa y su forma organizativa particular, mediante la responsabilidad y el auto-control, o lo que ha venido en llamarse empoderamiento. Las consecuencias de esta estrategia empresarial están dadas por una intensificación de los esfuerzos dedicados al trabajo gracias a su sistematización, seguimiento, evaluación (entre ella la de competencias) y mayor control, junto con una supuesta mayor autonomía del trabajador.

Relaciones de empleo. Diversos cambios se han presentado en las dinámicas del mercado laboral y las estrategias empresariales, tales como un aumento del perfil educativo exigido para el primer enganche o para el cambio de empresa, auge en las actividades de servicios, menor presencia del contrato a término indefinido, precarización bajo diferentes modalidades de contratación (temporal, a tiempo parcial, por prestación de servicios sin relación laboral, tercerización) disminución de la cobertura y la calidad de cubrimiento de la seguridad social y una mayor ingerencia de la mujer en los mercados de trabajo, pero aun en condiciones de desigualdad frente a los trabajadores varones en cuanto a sus condiciones de contratación y remuneración. Todo ello conlleva la profundización de la segmentación del empleo entre diversas calificaciones y distribución desigual entre sectores de la actividad económica en donde, por ejemplo, la mayor presencia femenina se da en actividades particulares como la de los centros de llamadas.

También se han establecido esquemas de remuneración variable de acuerdo con el cumplimiento de metas individuales y colectivas, lo que aumenta la presión que se ejerce sobre los trabajadores, no solamente por 
parte de la empresa, sino también de los colegas, para que se mantengan los máximos niveles de rendimiento, de los que depende la ganancia de las empresas y a la que se vincula el ingreso de los trabajadores, bajo la forma de complementos salariales o bonos por productividad, cumplimiento de metas, disminución de desperdicios, tiempos y movimientos.

Finalmente, bajo el nuevo modelo los mercados internos de trabajo, constituidos por aquellos mecanismos de ascenso en la empresa por méritos, antigüedad, cooperación o aportes a la innovación, tienden a desaparecer, en la medida que la flexibilidad y nuevas formas de contratación valoran menos los factores ligados a la experiencia.

Relaciones industriales. Después del auge del patrón de acumulación fordista, en donde los sindicatos se constituían en interlocutores válidos de las empresas, el auge neoliberal y las recientes transformaciones del patrón de acumulación introducen profundas modificaciones a la relación entre la empresa y sus trabajadores, en tanto los sindicatos son vistos como trabas para el buen funcionamiento de los mercados trabajo y la determinación de los salarios que, de acuerdo con los postulados de la teoría neoclásica, garanticen el pleno empleo. Por esto, en la óptica neoliberal los esquemas de organización de los trabajadores vienen a ser, junto con las regulaciones estatales, los principales culpables del desempleo, por lo que se trata de debilitarlos, cuestión que es facilitada en el nuevo patrón con sus principios organizativos y las exigencias de las nuevas tecnologías introduciendo, además, una profunda diferenciación entre los trabajadores sindicalizados y los no, con alto beneficio para estos últimos.

Significado para los trabajadores

Todos estos cambios tienden a fortalecer el ideal neoliberal de la individualización de las relaciones laborales, como pilar para el correcto funcionamiento del "mercado de trabajo", que se complementa con la modificación de las políticas sociales, con el desmonte del Estado bienestar, estableciendo una nueva forma de relación entre la propia sociedad con el Estado y de este con el mercado. Así, la privatización de la educación, salud, pensiones y demás componentes del paquete de protección social implica que cada individuo debe hacerse cargo de su cubrimiento, extrayendo parte del salario recibido para sufragar sus correspondientes costos. En forma correspondiente, el esquema de aseguramiento (o gestión del riesgo) es 
presentado como el mejor sistema para atender los "siniestros" que ocurran al trabajador en su salud o retiro del trabajo, puesto que al centralizar los aportes (primas de seguro) podrá atender las calamidades que se ocurran, lo que es esgrimido como argumento para justificar los inmensos recursos financieros centralizados por las empresas que conforman el sistema de seguridad social.

Los anteriores cambios en el patrón de acumulación, las nuevas exigencias al mundo del trabajo y la reconfiguración de las relaciones laborales, no significan nada más que una mayor alienación del trabajo, así se reclame la autonomía e independencia como sus mayores logros. Esto se hace patente en el afán por la individualización, que significa la estrategia del sálvese quien pueda y una progresiva desvalorización del trabajo y del mismo ser humano, bajo el discurso de que en la entrada a la "sociedad del conocimiento" el trabajador se desprende de las rutinarias labores manuales ganando su papel en tanto trabajador intelectual.

\section{COLOMBIA - MANIFESTACIONES EN LA COYUNTURA}

Las repercusiones de la crisis en curso han sido profundas para la población global y en particular para los trabajadores. En Colombia, la crisis tampoco es un fenómeno coyuntural ni meramente el reflejo o contagio de las manifestaciones financieras en el contexto global (1). En la coyuntura, a pesar de que durante largo tiempo se sostuvo por las autoridades económicas que el país estaba blindado frente a posibles contagios, el agravamiento de la crisis de largo plazo que sufre el país se ha sentido en variados frentes. Es de tener en cuenta que al igual que con el anterior modelo de acumulación fordista, la adopción del nuevo modelo ha sido parcial por parte del empresariado, en la medida en que si bien desde los años 80 se comenzaron a introducir los componentes generales dictados por el enfoque neoliberal de disminución del Estado, privatización y liberalización de los mercados, los elementos del modelo productivo escasamente han sido adoptados. Aun así, sus incidencias en el mundo laboral sí se han manifestado, como se ilustra en las siguientes secciones.

\section{Adopción del paradigma tecnoeconómico}

Varias encuestas aplicadas a las empresas colombianas desde comienzos de los noventas muestran la manera como se han venido introduciendo 
lentamente las nuevas tecnologías en los diferentes procesos, que en general han tenido baja utilización en objetivos estratégicos de la producción y el énfasis ha sido puesto en el manejo operativo y de coordinación, por lo que se pueden considerar más como un elemento de soporte a los cambios que la causa de estos mismos $(5,6)$.

En cuanto al supuesto aumento de la autonomía del trabajador en los procesos, las nuevas tecnologías se han buscado más como elemento de control y para facilitar el recambio de empleados jóvenes por los trabajadores antiguos, lo cual ha reforzado el hecho de que la vida laboral promedio de los colombianos sea de 35 años. La flexibilidad en sus diversas manifestaciones y la tercerización de funciones han obtenido el debilitamiento de las organizaciones sindicales.

Un análisis de la situación actual de las relaciones laborales en el país ilustra lo anterior.

Relaciones de trabajo

La forma como han sido introducidas las tecnologías modernas a las organizaciones productivas muestran las consecuencias generales, como ha sido ilustrado en un reciente trabajo adelantado por Anita Weiss y colaboradores (5). La consecuencia fundamental ha sido la intensificación del trabajo, mayor exigencia de responsabilidad individual, intensificación del control directo, mientras los fenómenos de calificación y descalificación se dan simultáneamente, en sentido contrario a lo afirmado por los propagandistas del nuevo modelo, que pregonan la exigencia de mayor calificación laboral.

Relaciones de empleo

Pese a las notorias tasas de crecimiento de la economía en años recientes, las condiciones estructurales de desempleo y pobreza no se vieron modificadas y más bien, han sido agravadas en la coyuntura. En esta dirección, lo que los economistas consideran la tasa de desempleo de largo plazo o estructural -aquélla por debajo de la cual es difícil caer- subió de nivel, pues del $8 \%$ que se calculaba en los años noventa, llegó al $11 \%$ en la presente década, con tendencia al alza en el año en curso, lo que hace que el país se ubique como el segundo con desempleo más alto de los países similares en la región. 
Este mayor desempleo no es coyuntural ni mero producto de la crisis. De tiempo atrás, y particularmente en los años del gobierno Uribe, se manifiesta como resultado del modelo económico aplicado, que conduce a la reprimarización del aparato productivo, acorde con una inserción en el mercado mundial basada en la exportación de productos primarios y donde la innovación tecnológica no es un proceso endógeno estructurado a la economía de modo que el componente de ciencia y tecnología que caracteriza el patrón de acumulación de referencia mundial no es prioridad en la agenda de desarrollo. Al contrario, en el aparato productivo se tiende a reemplazar el trabajo por mayor capital incorporado, lo que ha sido reforzado por la política aplicada, que brinda fuertes exenciones y subsidios al capital, con lo cual en los dos últimos años ha tenido un fuerte aumento la relación incremental capital - producto, que nos dice que en cada unidad de producto obtenido viene aumentando el componente de capital y, por consiguiente, reduciéndose el de trabajo (7).

A la par con el incremento del desempleo, se ha mantenido en el tiempo el desmejoramiento de la calidad del empleo. La desagregación de los procesos mediante la subcontratación ha venido profundizándose en las empresas de todos los sectores, con lo cual se sustituyen trabajadores directos, eliminando parte de la plantilla de personal. Uno de los esquemas de más rápido auge en este tipo de contratación es el de las cooperativas de trabajo asociado, buena parte de las cuales se organizan por las mismas empresas para romper capacidad de negociación laboral y algunas simplemente engañan a sus afiliados y no asumen los compromisos de afiliación a la seguridad social. Finalmente, y de manera sobresaliente en el sector oficial, abunda la contratación temporal mediante la figura de "órdenes de prestación de servicios", que no generan ninguna relación laboral con los trabajadores, debiendo asumir éstos el pago de su seguridad social a partir de un ingreso de carácter integral que toma la forma de honorarios.

Es así como de los 2,4 millones de empleos creados en los últimos seis años, más de una tercera parte corresponde a contratos temporales o de tiempo parcial, con la consecuente evasión de la protección social a cargo de las empresas. Aun más, cerca de 1,5 millones de los trabajadores que se ocuparon durante los últimos seis años lo hicieron en la modalidad de cuenta propia, categoría supremamente precaria por carecer de condiciones estables de ingreso. Con esta cifra, ya asciende a 7,9 millones el total de trabajadores que se desempeñan bajo esta modalidad. 
Como consecuencia, la informalidad laboral se ha mantenido en niveles cercanos al $60 \%$, manifestándose con más fuerza en el empleo femenino.

En la empresa, la utilización de las nuevas tecnologías ha permitido una definición más exacta de la cantidad de personas a emplear, junto con la disminución relativa de personal de planta con contrato a término indefinido y el cambio de perfil de las personas ocupadas (jóvenes con mayores niveles de calificación). Todo esto permite la coexistencia de personas con diversos tipos de contrato laboral en una misma empresa: contratos a término indefinido, temporales, contratos a término fijo que pueden durar 15 años, contratos de tiempo parcial o reducido, contratos a través de cooperativas de trabajo asociado, ya sea como modalidad de empleo o para realizar labores especializadas, contratos por prestación de servicios (individuales o colectivos), contratación de servicios que se tercerizan pero que se ejecutan en las instalaciones de las empresas contratantes. Como resultado, se evidencia una profunda erosión de los mercados internos de trabajo (5)

Relaciones industriales

Los procesos de negociación individual y colectiva entre las empresas y los trabajadores, así como entre las empresas y los sindicatos, pero también entre los trabajadores y los sindicatos se han deteriorado de manera profunda en el país ante la disminución de la porción de trabajadores sindicalizados, la eliminación de los contratos a término indefinido y la no negociación de convenciones colectivas a cambio del otorgamiento de pactos colectivos, lo que reproduce el modelo paternalista adoptado en el manejo del Estado a la dirección de las empresas. El objetivo final y alcanzado en buen grado, es debilitar la capacidad de movilización del conjunto laboral, mediante la diferenciación de trabajadores y una gran diversidad de relaciones de empleo con la diferenciación de los regímenes laborales, todo lo cual causa un deterioro del poder de negociación, expresión de la tendencia hacia la individualización de las relaciones laborales.

Ingresos, pobreza e iniquidad social

El resultado neto de este modelo a la colombiana es una redistribución regresiva de la riqueza bajo la forma de un desplazamiento de la participación de los trabajadores en el producto generado a favor de las ganancias del capital, como se ilustra en la Figura 1, donde es claro que 
los excedentes generados con el crecimiento quedaron en manos de los propietarios de los activos, pues la brecha en la apropiación del producto generado se hace más amplia a favor de los excedentes empresariales y en contra de los trabajadores.

Figura 1. Relación ganancia-salario, 2002-2007

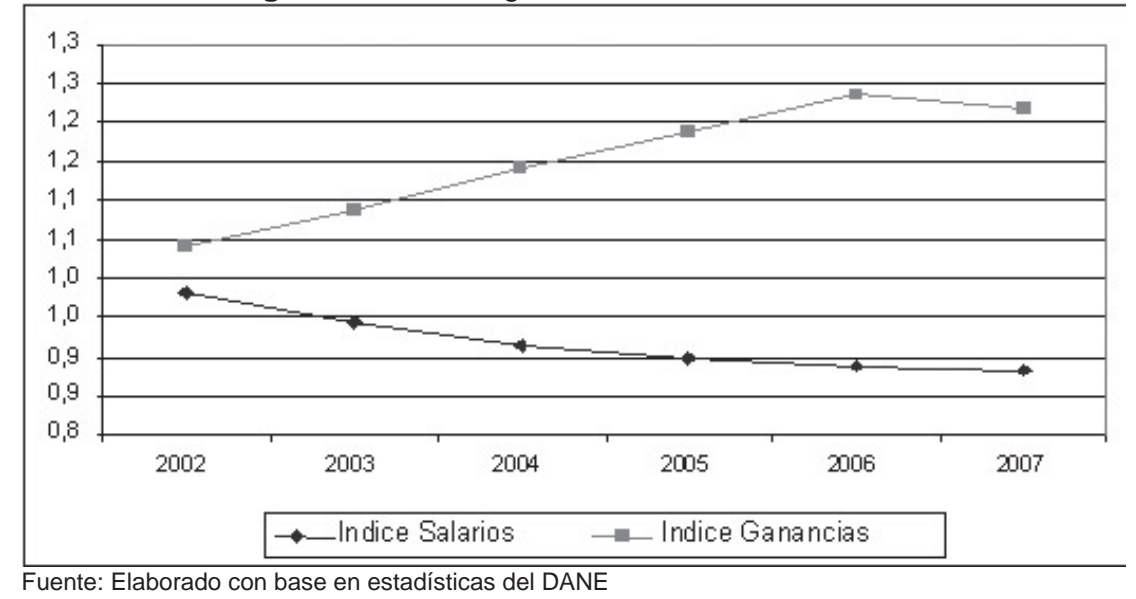

Lo anterior no es más que el resultado lógico de la disminución en el salario real observada en los últimos años, en la medida en que la evolución de los salarios se ha alejado en caída libre con respecto al aumento de la productividad laboral, como se puede apreciar en la figura 2.

Figura 2. Relación salario - productividad, 2001-2006

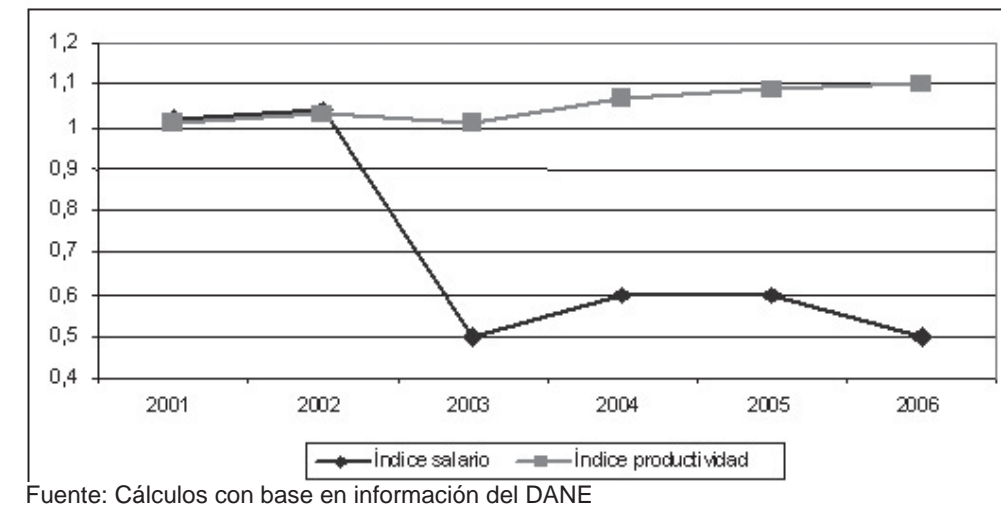


Todo ello se manifiesta en el conjunto de los indicadores sociales que muestran cómo el nivel de pobreza, de acuerdo con las cifras oficiales de 2008, abarca el $46 \%$ de la población, (ligeramente menor que el de 1995 que se ubicaba en 49,5\%, cuando el país creció al 5,2 \%), la que sobrevive con menos de dos dólares al día. Pero como la población creció, lo dramático es que hoy en día hay más pobres que una década atrás, lo que desmiente en sí la afirmación oficial de que la pobreza se haya reducido.

Lo que es peor, la pobreza rural es la más dramática $(65,2 \%)$ reflejado en el hecho de que existen 655000 hogares sin tierra y 2,2 millones de propietarios de micro y minifundios que, para lo peor de su situación, están siendo expulsados de sus territorios en forma tal que hay ya alrededor de 4 millones de personas desplazadas. Todo esto se refleja en el hecho de que la indigencia medida a escala nacional haya llegado al 17,8 \% de la población, mientras la rural es de un escandaloso 32,6 \%. El resultado, mayor concentración en la gran propiedad y legitimación del despojo a campesinos, afro descendientes e indígenas.

La síntesis de esta grave crisis la muestra la alta desigualdad que refleja un Gini nacional de 0,59 , uno de los más altos del mundo (8).

\section{Seguridad social}

La adopción de los rasgos generales del nuevo modelo de acumulación en Colombia, bajo los principios de la individualización en el mercado, ha dado origen a un sistema caracterizado por la segmentación, que genera inequidades en la calidad de vida y la salud de la población, estableciendo segregaciones de acuerdo con la capacidad de pago de los individuos y las familias, entre pobres, ingresos medios y ricos, para quienes se corresponden los regímenes en salud de subsidiados, cotizantes y prepagados.

La aplicación parcial del nuevo modelo reforzó la crisis prolongada que atraviesa el país, una de cuyas manifestaciones más graves se da en la protección social, que refleja no solo la precariedad en el mundo laboral sino la gran desigualdad social y las consecuencias del conflicto en sus múltiples manifestaciones de marginalidad, desplazamiento y miseria. Las cifras oficiales del DANE muestran que de los 18 millones de personas ocupadas laboralmente en el país, la mitad lo hace bajo una cualquiera de las categorías salariales, y de esos nueve millones tan solo hay seis 
millones activos en el sistema de salud y pensiones, quedando los restantes tres sin cobertura o en el difuso régimen subsidiado. Éstos últimos vienen a sumarse a los trabajadores por cuenta propia ya señalados, cuya situación de seguridad también es bastante precaria. Situación que se agrava en la cobertura de los riesgos profesionales, existente tan solo para una tercera parte de las personas ocupadas, con mayor vulnerabilidad por parte de los trabajadores informales (9). En resumen, sería una tercera parte del universo de personas ocupadas en Colombia la que se encuentra en situación de indefensión social y que, como bien se sabe, con sus familias como receptoras de todas las consecuencias, al igual que las comunidades en las que ellas se inscriben.

Caso muy similar es la situación de las personas que de acuerdo con la legislación colombiana tendrían derecho a disfrutar de una pensión de jubilación y que ya alcanzan la cifra de cuatro millones y medio. De ellas, tan solo un millón se encuentran devengando un ingreso de pensión y el resto califica en la categoría de cuenta propia o dependientes del ingreso de algún miembro de la familia. $\mathrm{Y}$ en concordancia con lo enunciado arriba sobre precariedad laboral, tan solo una tercera parte de los trabajadores activos está registrada en el sistema de pensiones. Con la inestabilidad, precariedad e incertidumbre del mercado laboral las esperanzas de alcanzar una pensión son más inciertas para los trabajadores colombianos.

\section{FORMAS DE ENFRENTAR LA COYUNTURA}

Con la crisis y sus variadas manifestaciones, el mundo del trabajo enfrenta una encrucijada de grandes proporciones, tanto en el plano internacional como en el local. La consolidación del nuevo patrón de acumulación amenaza con dejarlo aun más debilitado que antes de la emergencia de los síntomas financieros de la crisis. La segmentación, precariedad y desprotección laboral implican que las viejas estrategias de reivindicación sindical tienen muy pocas posibilidades de lograr relevancia para dar alcance social a las demandas de los trabajadores. El reto, entonces, es ensayar nuevas formas de organización y de movilización social, que a la vez que hagan resistencia a la manera regresiva de implantar el nuevo modelo, permita ganar consenso para que la sociedad asuma la necesidad de adoptar nuevos esquemas de desarrollo que ataque los problemas de largo plazo y sea sostenible en lo social, lo económico y lo ambiental, con la premisa de alcanzar el bienestar económico y social, logrando pleno empleo y eliminando la pobreza (10). 
La estructuración de una estrategia para avanzar en esos propósitos pasa por comprender y hacer visibles las reales causas de la crisis y la complejidad de factores que están actuando en contra de la construcción de condiciones que permitan el buen vivir a toda la población, para lo cual se debe:

- Precisar los fenómenos que caracterizan la crisis estructural generalizada y de largo plazo.

- Identificar los actores y factores impulsores de la actual situación en los diferentes niveles de decisión: sitio de trabajo, planos local y nacional.

- Presionar por la modificación del orden económico mundial y el cambio de paradigma en la definición de políticas globales y nacionales que pongan la equidad en el mismo plano del crecimiento económico.

- Incluir en la movilización social a sectores afectados pero que no tienen posibilidad de articularse debido a la estrategia global del capital.

- Plantear y presionar por la modificación del régimen de seguridad social imperante por uno de protección social integral que posibilite los objetivos de equidad y pleno empleo.

- Definir e impulsar, en los planos local y nacional, los componentes y adopción de un nuevo modelo que transforme significativamente la manera como se ha dado manejo a las cuestiones económicas y sociales, en torno a propósitos comunes de crecimiento y equidad como entronque de un acuerdo que concite a los actores sociales en la búsqueda de sus objetivos de bienestar común

\section{REFERENCIAS}

1. Zerda A. La actual crisis del capitalismo. Crisis de la economía y crisis de la teoría económica. Facultad de Ciencias Económicas, Universidad Nacional de Colombia, Bogotá, D. C.; Junio 2009.

2. Boutang YM. Nouvelles frontières de l'économie politique du capitalisme cognitif. Revue éc/arts. 2007: 3: 1-36.

3. Zerda A. Apertura, nuevas tecnologías y empleo, Bogotá, D. C. Fescol; 1992.

4. Zerda A. Innovación tecnológica en el nuevo modelo productivo. Bogotá, D. C. ILSA; 2009.

5. Amézquita $C$ Panorama de la sociedad de la información en América Latina (2000-2007). Revista Facultad de Ciencias Económicas, XVII (2): 151-170. 
6. Zerda A. Las encuestas de desarrollo tecnológico en Colombia. Bogotá, D. C. Facultad de Ciencias Económicas, Universidad Nacional de Colombia; 2008.

7. Moreno A [Internet]. Economía y Seguridad Democrática. ¿Quiénes ganan y quiénes pierden con Uribe? Revista electrónica Razón Crítica. Disponible en : http:// www.razonpublica.com/index.php?option=com_content\&task=view\&id=571\&ltem id=165 Consultado: 17 de agosto 2009.

8. DANE. Misión para el Empalme de las Series de Empleo, Pobreza y Desigualdad. Bogotá, D. C.; agosto de 2009.

9. Hernández M, Restrepo DI, Ochoa D. Solidaridad en serio: hacia una protección social universal en Colombia. En: Cárdenas ME, Suárez, A (Coords.) Bases de una política económica para la productividad, el empleo y la distribución del ingreso. Bogotá, D. C. Fescol; 2009.

10. Zerda A. Mercado interno: base de la acumulación productiva con equidad. En: Cárdenas ME, Suárez, A (Coords.) Bases de una política económica para la productividad, el empleo y la distribución del ingreso. Bogotá, D. C. Fescol; 2009. 